\title{
Distribution and Molecular Characterization of Functional Class 2 Integrons in Clinical Proteus mirabilis Isolates
}

\author{
Wenjun Lu'*, Quedan Qiü ${ }^{2} *$, Keda Chen ${ }^{2}$, Rongqing Zhao ${ }^{2}$, Qingcao $\mathrm{Li}^{2}$, Qiaoping Wu ${ }^{2}$ \\ 'Intensive Care Units of Ningbo Medical Centre Lihuili Hospital, Ningbo University, Ningbo, Zhejiang Province, People's Republic of China; ${ }^{2}$ Clinical \\ Laboratory of Ningbo Medical Centre Lihuili Hospital, Ningbo University, Ningbo, Zhejiang Province, People's Republic of China \\ *These authors contributed equally to this work \\ Correspondence: Qingcao Li; Qiaoping Wu, Tel +86-574-55835786, Fax +86-574-5583578I, Email lqc_lab@I63.com; Ihlyywqp@।63.com
}

\begin{abstract}
Background: Integrons are the main mode of horizontal transmission of drug-resistance genes and are closely related to drug resistance in clinical bacteria. In this study, the distributions of class 1, 2, and 3 integron gene cassettes were investigated in 150 Proteus mirabilis (P. mirabilis) isolates from patients, and molecular characterization of functional class 2 integrons was further analyzed.

Methods: Class 1, 2, and 3 integrons were screened by polymerase chain reaction (PCR) in 150 clinical P. mirabilis isolates. The variable regions of the integrons were determined by restriction analysis and sequencing. Internal stop codons mutations in class 2 integrons and their common promoters were also determined by sequencing. Enterobacterial repetitive intergenic consensus polymerase chain reaction (ERIC-PCR) was used to analyze the phylogenetic relations of class 2 integron-positive isolates.

Results: Class 1 integrons were detected in 69 (46\%) of 150 P. mirabilis isolates, and six different gene cassette arrays were detected, with the most prevalent being $d f r A 32$-aadA2. Class 2 integrons were detected in $61(40.7 \%)$ of 150 P. mirabilis isolates, and three different gene cassette arrays were detected, including sat2-aadA1, which was detected for the first time in a class 2 integron. Nearly similar ERIC-PCR fingerprinting patterns were detected in 45 (73.8\%) of 61 class 2 integron-positive isolates. The functional class 2 integron was detected in three P. mirabilis isolates having the same gene cassette, dfrA1-sat2-aadA1, in the variable region and four novel open reading frames with unknown functions. Same PintI2 and Pc promoters were detected in these three functional class 2 integron isolates, as was found in other class 2 integron isolates. However, these three strains did not totally show identical homology and drug sensitivity.
\end{abstract}

Conclusion: Although functional class 2 integrons have low distribution and relatively conserved molecular characteristics, they can still form clinical dissemination and drug resistance expression.

Keywords: Proteus mirabilis, integrons, premature stop codon, promoter regions, antibacterial drug resistance

\section{Introduction}

Integron is a gene unit that acquires exogenous drug resistance genes and allows their expression in bacteria. It is an important factor in the horizontal transmission of bacterial drug resistance. With the effect of integrase, bacteria capture and express foreign genes expressing drug resistance through the integron-gene cassette system, manifesting multi-drug resistance and pan-drug resistance. ${ }^{1-3}$ The resistance gene cassettes are spread in two ways: one is transfer between integrons via specific recombination sites on the integron, the other is the gene cassette located on the integron forming a circular gene cassette under the catalytic shearing of the integrase, and then horizontal transfer by transduction or transformation between various bacteria. ${ }^{4}$ The resistance genes currently found in the integrons include mediating betalactamases, aminoglycosides, trimethoprim, streptomycin, streptomycin, chloramphenicol, rifampicin, erythromycin, and quaternary ammonium compound disinfectants, and more novel drug resistance genes are constantly being discovered. ${ }^{5,6}$ 
Moreover, the same gene cassette composition and arrangement sequence can be detected among different strains, which further proves that integrons have the ability to capture, carry and reorganize gene cassettes can also spread among different bacteria. ${ }^{7}$ Based on the first-level structure of integrase proteins, integrons have been classified into four types. Class 1 integron is the most widely distributed in clinical strains and has been studied in-depth. Class 2 integron has the second-highest isolation rate, which was translated to truncated integrase proteins because of an early stop codon TAA after the 178th amino acid. It was considered a defective integron as it cannot integrate and cut resistance gene cassettes. ${ }^{8,9}$ Therefore, studies on class 2 integrons were limited. However, the discovery of functional class 2 integrons has changed the traditional view that class 2 integrons had no functional. ${ }^{10,11}$ In this study, we screened class 1, 2, and 3 integrons in 150 clinically isolated strains of $P$. mirabilis, analyzed their drug resistance, and confirmed the distribution and structure of functional class 2 integrons.

\section{Materials and Methods}

\section{Bacterial Strains}

One hundred and fifty strains of $P$. mirabilis were isolated from non-repetitive clinical specimens such as sputum, midstream urine, blood, and traumatic secretions from January 2018 to December 2020 in our hospital and were used as experimental strains. Engineered strains of Escherichia coli DH5a (class 1, 2, and 3 integron negative control strains), $P$. mirabilis 47437 (class 1 and 2 integron positive control strains), Serratia marcescens 37586 (class 3 integron positive control strain), and functional class 2 integron positive strains were provided by Dr. Quhao Wei. ${ }^{11}$

\section{Identification and Antimicrobial Susceptibility Testing of Bacteria}

All isolates were identified by Vitek-2 Compact (BioMe 'rieux, Marcy-l'Etoile, France). Several antibiotics were selected as representative drugs. These antibiotics were tested for antimicrobial susceptibility using the disk diffusion method according to the Clinical and Laboratory Standards Institute guidelines. ${ }^{12}$ The antibiotics chosen were amikacin, gentamicin, tobramycin, and sulfamethoxazole/trimethoprim (SMZ/TMP). Escherichia coli ATCC25922 was used as the control for antibiotic resistance.

\section{Bacterial DNA Preparation and Integron Analysis}

Total genomic DNA was isolated from stationary-phase cultures that were grown overnight in Luria-Bertani broth (Oxoid, UK) using the EZ-10Spin Column Bacterial Genomic DNA MiniPreps Kit (Bio Basic Inc, Canada) according to the manufacturer's instructions. Primers used in this study are listed in Table $1 .{ }^{11,13}$ For PCRs, rTaq DNA polymerase (TaKaRa, Japan) was used to screen integrons, while LA Taq DNA polymerase (TaKaRa, Japan) was used in PCRs to amplify the variable regions of integrons. Considering variable regions of class 1 and class 2 integrons, same-sized amplicons were compared by restriction analysis using HinfI (TaKaRa, Japan) and Cfr13I (Fermentas, Canada) restriction endonucleases, respectively. Primer walking was used to sequence at least one representative of each type of amplicon, as described previously. Sequence analysis was conducted using Vector NTI Advance 11 (Invitrogen, USA).

\section{Determination of the Internal Stop Codon Mutation and Common Promoter in the int/2 Gene}

In intI2 (coding for a functional IntI2 integrase) gene-positive strains, primer intI2F combined with $d f r A 1-R$ or sat2-R (Table 1) were used to amplify the intI2 gene together with attI2 and the common promoter (PintI2 and Pc2), which is located at the attI2 site and can drive the transcription of the downstream intI2 and gene cassette in the variable region of class 2 integrons. PCR products were sequenced directly, and the internal stop codon mutations in the intI2 genes, as well as the types of common promoters of class 2 integrons, were determined manually according to the obtained sequences.

\section{Typing of int/2-Positive $P$. mirabilis by ERIC-PCR}

The phylogenetic relations of intI2 gene-positive P. mirabilis were analyzed using ERIC-PCR with primer ERIC2 (Table 1) as described previously. ${ }^{14}$ The amplification conditions were as follows: denaturation for 4 min at $94^{\circ} \mathrm{C} ; 40$ 
Table I The Primers and Their Sequences for Integron Screening

\begin{tabular}{|c|c|c|c|}
\hline Primer & Target Gene or Region & Sequence $\left(5^{\prime} \rightarrow 3^{\prime}\right)$ & Reference \\
\hline intF & intll & CCAAGCTCTCGGGTAACATC & {$[\mathrm{II}]$} \\
\hline$P 2 R$ & & GCCCAGCTTCTGTATGGAAC & {$[11]$} \\
\hline int $12 F$ & int $/ 2$ & GTAGCAAACGAGTGACGAAATG & {$[\mathrm{II}]$} \\
\hline int $/ 2 R$ & & CACGGATATGCGACAAAAAGGT & [1I] \\
\hline $\operatorname{int} / 3 F$ & int/3 & AGTGGGTGGCGAATGAGTG & [13] \\
\hline $\operatorname{lnt} / 3 R$ & & TGTTCTTGTATCGGCAGGTG & [13] \\
\hline $5 C S$ & Class I integron variable region & GGCATCCAAGCAGCAAG & {$[\mathrm{II}]$} \\
\hline $3 C S$ & & AAGCAGACTTGACCTGA & {$[\mathrm{II}]$} \\
\hline INF2 & Class 2 integron variable region & TGGGTGAGATAATGTGCATC & {$[11]$} \\
\hline INB2 & & TCGAGAGAGGATATGGAAGG & {$[\mathrm{II}]$} \\
\hline$d f r A I-R$ & Class 2 integron att $/ 2$ region att 12 region & AGGAGCTGTTCACCTTTGGCAC & {$[11]$} \\
\hline sat2-R & Class 2 integron att 2 region att 12 region & TCGATGTCGATCGTCGATAAG & {$[\mathrm{II}]$} \\
\hline ERIC2 & Integron homology & AAGTAAGTGACTGGGGTGAGCG & {$[11]$} \\
\hline
\end{tabular}

cycles of $40 \mathrm{~s}$ at $94^{\circ} \mathrm{C}, 1 \mathrm{~min}$ at $40^{\circ} \mathrm{C}$ and $5 \mathrm{~min}$ at $72^{\circ} \mathrm{C}$; and a final extension step at $72^{\circ} \mathrm{C}$ for $10 \mathrm{~min}$. The products were separated by electrophoresis on a $1 \%$ agarose gel containing ethidium bromide $(0.5 \mu \mathrm{g} / \mathrm{mL})$ in $1 \times$ Tris/acetate/EDTA buffer for $1 \mathrm{~h}$ at $80 \mathrm{~V}$. The generated fingerprints were compared visually.

\section{Statistical Analysis}

The experimental data were analyzed using SPSS v19.0 (IBM, Armonk, NY). Antibiotic resistance was compared by the Chi-square test. Comparisons were considered significant if $\mathrm{p}<0.05$.

\section{Results}

\section{Integrons and Their Gene Cassettes in P. mirabilis Isolates}

Class 1 integrons were detected in $69(46 \%)$ of 150 P. mirabilis isolates. In these 69 class 1 integron-positive strains, variable regions were successfully amplified in 65 isolates. In total, six different gene cassette arrays were detected (Table 2). The most prevalent gene cassette arrays were $d f r A 32$-aadA2 and dfrA32-ereA1-aadA2, which were detected in 40 and 13 isolates.

Class 2 integrons were detected in $61(40.7 \%)$ of 150 P. mirabilis isolates. Among these, 39 isolates were also positive for class 1 integrons. Variable regions of class 2 integron were successfully amplified in 50 isolates. In total, three different gene cassette arrays were detected (Table 2). The most prevalent of these gene cassette arrays was $d f r A 1$ sat2-aadA1, which was detected in 47 isolates. The cassette $d$ frA1-catB2-sat2-aadA1 was detected in two isolates, and sat2-aadA1 was detected in one isolate. As far as we know, it is the first report of the sat2-aadA1 cassette in a class 2 integron.

The last gene cassette of the variable region of class 2 integrons obtained in this study was aadA1, which was found to be followed by four novel open reading frames (ORFs). These four newly detected ORFs were temporarily named $y b e A, y b f A, y b f B$, and $y b g A$ in this study. Sequence comparisons were carried out using the Basic Local Alignment Search Tool program. However, no matching sequence was retrieved with sequences of these four ORFs, making it difficult to identify the functions of these ORFs. The structural characteristics and translation directions of the variable region gene cassettes are presented in Figure 1A.

\section{Comparisons of Resistance Phenotypes}

The results of the Chi-square test showed that the antibacterial resistance rates of the integrase positive strains to gentamicin, tobramycin, and cotrimoxazole were significantly higher than the integrase negative strains $(\mathrm{P}<0.01)$. The rates of resistance to gentamicin and tobramycin in both class 1 and 2 integrase positive strains were significantly higher than those in class 1 or 2 integrase positive strains alone and integrase positive cassette without detection of related genes 
Table 2 The Distribution of Class I and 2 Integrons and Gene Cassettes in 150 Strains of Clinically Isolated Proteus mirabilis

\begin{tabular}{|c|c|c|c|c|c|}
\hline Number & intll & Variable Region of Class I Integron & Int/2 & Variable Region of Class 2 Integron & ERIC Type (Number) \\
\hline 59 & - & ND & - & ND & ND \\
\hline 3 & + & $\_^{\mathrm{a}}$ & - & ND & ND \\
\hline 19 & + & dfrA32-aadA2 & - & ND & ND \\
\hline 5 & + & dfrA32-ereAI-aadA2 & - & ND & ND \\
\hline 2 & + & aadB-aadA2 & - & ND & ND \\
\hline I & + & CatB3 & - & ND & ND \\
\hline 11 & + & $d f r A 32-a a d A 2$ & + & $-^{\mathrm{a}}$ & $A(9), B(2)$ \\
\hline 22 & - & ND & + & $d f r A I$-sat2-aadA I-(ybeA-ybfA-ybfB-ybgA) ${ }^{b}$ & $A(18), B(3), C(I)$ \\
\hline I & + & $\_^{a}$ & + & $d f r A I$-sat2-aadA I-(ybeA-ybfA-ybfB-ybgA) ${ }^{b}$ & $A(I)$ \\
\hline 9 & + & dfrA32-aadA2 & + & $d f r A I$-sat2-aadA I-(ybeA-ybfA-ybfB-ybgA) ${ }^{b}$ & $A(7), B(2)$ \\
\hline 8 & + & dfrA32-ereAI-aadA2 & + & $d f r A I$-sat2-aadA I-(ybeA-ybfA-ybfB-ybgA) ${ }^{b}$ & $A(5), B(2), D(I)$ \\
\hline 7 & + & $\operatorname{aadB}-a a d A 2$ & + & $d f r A I$-sat2-aadA I-(ybeA-ybfA-ybfB-ybgA) ${ }^{b}$ & $A(3), C(2), D(2)$ \\
\hline 1 & + & dfrA32-aadA2 & + & $d f r A 1$-catB2-sat2-aadA I-(ybeA-ybfA-ybfB-ybgA) ${ }^{b}$ & $A(I)$ \\
\hline I & + & $d f r A \mid 2$-orfF-aadA2 & + & dfrAl-catB2-sat2-aadA I-(ybeA-ybfA-ybfB-ybgA) ${ }^{b}$ & $E(I)$ \\
\hline I & + & $\operatorname{aadA2}$ & + & sat2-aadA I-(ybeA-ybfA-ybfB-ybgA) ${ }^{b}$ & $A(I)$ \\
\hline
\end{tabular}

Notes: aPCR failed to amplify variable region that was resistant to gene cassette. bybeA, ybfA, ybfB, and ybgA are four open reading frames. Whether it is a drug-resistant gene cassette and its function remain to be determined. Abbreviation: ND, not detected. 
A

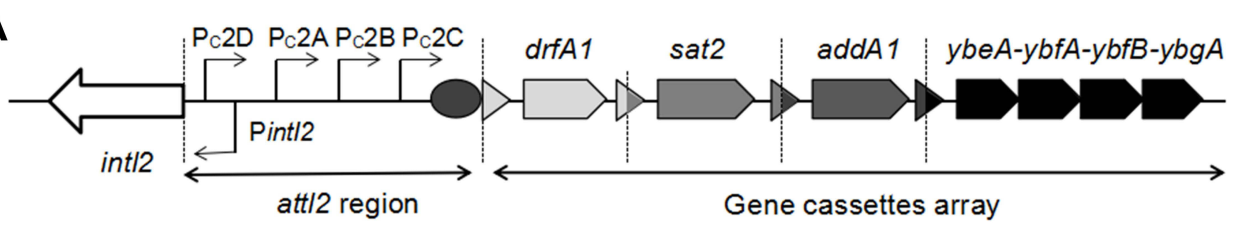

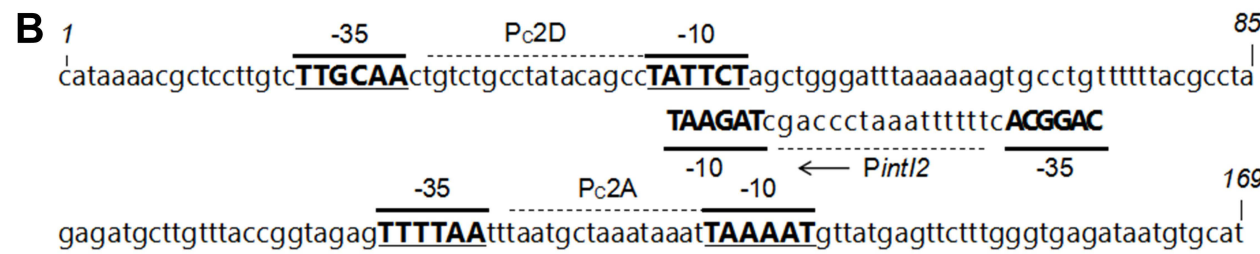

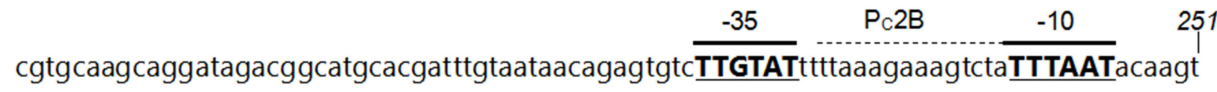

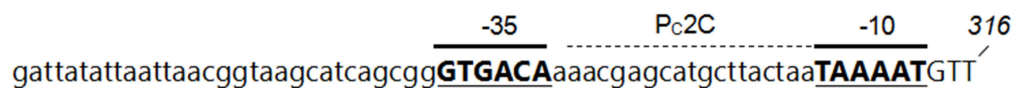

Figure I The functional class 2 integrons. (A) General structure of the functional class 2 integrons: Arrows indicate the coding sequences with the gene name above, triangles and circles are attC and attl recombination sites, respectively. The attl 2 region and gene cassette array are indicated. Dotted vertical bars separate each gene cassette. (B) Nucleotide sequence of the attl2 region. The -35 and -10 elements of the Pc2 promoters are written in bold uppercase, and their names are indicated. The transcriptional mapped for Pint 12 is indicated by a Broken Arrow and bold uppercase. The position of several nucleotides is numbered (italics).

$(\mathrm{P}<0.01)$. The rates of resistance to SMZ-TMP in the class 1 integrase positive strains and in both class 1 and 2 integrase positive strains were $88.9 \%$ and $96.3 \%$, respectively, and were significantly higher than in those of class 2 integrase positive strains and integrase positive non-associated gene cassettes $(\mathrm{P}<0.01)$. There was no significant difference between groups in terms of the resistance to amikacin (see Table 3).

\section{Functional Class 2 Integrons in P. mirabilis Isolates}

Sequences analysis revealed functional class 2 integrons in three P. mirabilis isolates. Results showed that the internal stop codons in these intI2 genes were all mutated from TAA to the glutamine codon CAA (Figure 2), implying the presence of functional intI2 genes. As described previously, these three functional class 2 integrons had the same gene cassette of the variable region, containing dfrA1-sat2-aadA1 and four novel ORFs. Sequence analysis showed that the functional class 2 integron recombination site starts from attl2 to $316 \mathrm{bp}$ sequence downstream and was observed to be relatively conserved as other class 2 integrons ${ }^{15}$ (Figure 1B). The PintI2 transcription start site was mapped at position 43 upstream from the attI2 start codon, in agreement with previously class 2 integron inferred -35 and -10 elements (CAGGCA and TAGAAT, respectively, separated by 17 bp; Figure 1B). Four potential Pc2 promoters were detected (named here as Pc2A, Pc2B, Pc2C, and Pc2D; Figure 1B), within the attI2 region. These three functional class 2 integrons isolates were not identical in carrying class 1 integron and related antibiotic-resistant phenotypes (Table 4). Full gene sequencing revealed that these three functional Class 2 integrons are located on the $\operatorname{Tn} 7$ transposons and the host bacteria chromosome, as reported in previous studies. ${ }^{8}$

\section{Clonal Spread of Class 2 Integron-Harboring P. mirabilis}

The phylogenetic relations of 61 class 2 integron-positive P. mirabilis isolates were typed by ERICP-PCR, and five ERIC types were obtained according to the electrophoresis patterns (Table 2 and Figure 3). ERIC type A and B were found to have mainly 45 and 9 isolates, respectively. As shown in Figure 3, two of the three functional class 2 integron-positive $P$. mirabilis isolates were observed to be of ERIC type A, and they were the same as in other most class 2 integron-positive strains. Another functional class 2 integron-positive isolate was found to be of ERIC type B. These results showed that a trend of dissemination still exists, despite the low clinical distribution of functional class 2 integrons. 
Table 3 Comparison of Antimicrobial Resistance Rates of I50 Strains of Proteus mirabilis with Major Resistance Genes in Variable Regions (\%)

\begin{tabular}{|c|c|c|c|c|c|c|c|c|c|}
\hline \multirow[t]{2}{*}{ Group } & \multirow{2}{*}{$\begin{array}{l}\text { Number } \\
\text { of Strains }\end{array}$} & \multicolumn{2}{|c|}{ Amikacin } & \multicolumn{2}{|c|}{ Gentamicin } & \multicolumn{2}{|c|}{ Tobramycin } & \multicolumn{2}{|c|}{ SMZ-TMP } \\
\hline & & $R$ (n) & $\begin{array}{l}\text { Resistance } \\
\text { Rate }\end{array}$ & $R(n)$ & $\begin{array}{l}\text { Resistance } \\
\text { Rate }\end{array}$ & $R$ (n) & $\begin{array}{l}\text { Resistance } \\
\text { Rate }\end{array}$ & $R(n)$ & $\begin{array}{l}\text { Resistance } \\
\text { Rate }\end{array}$ \\
\hline intl/ positive & 27 & I & $3.7 \%$ & 13 & $48.2 \%$ & II & $40.7 \%$ & 24 & $88.9 \%$ \\
\hline int/2 positive & 22 & I & $4.6 \%$ & 9 & $40.9 \%$ & 5 & $22.7 \%$ & 9 & $40.9 \%$ \\
\hline int/l and int/2 positive & 27 & 2 & $7.4 \%$ & 23 & $85.2 \%$ & 22 & $81.5 \%$ & 26 & $96.3 \%$ \\
\hline $\begin{array}{l}\text { Integrase positive and no related } \\
\text { gene cassette detected }\end{array}$ & 15 & 0 & $0.0 \%$ & 6 & $40.0 \%$ & 3 & $20.0 \%$ & 6 & $40.0 \%$ \\
\hline Integrase negative & 59 & 0 & $0.0 \%$ & 2 & $3.4 \%$ & 0 & $0.0 \%$ & 6 & $10.2 \%$ \\
\hline
\end{tabular}




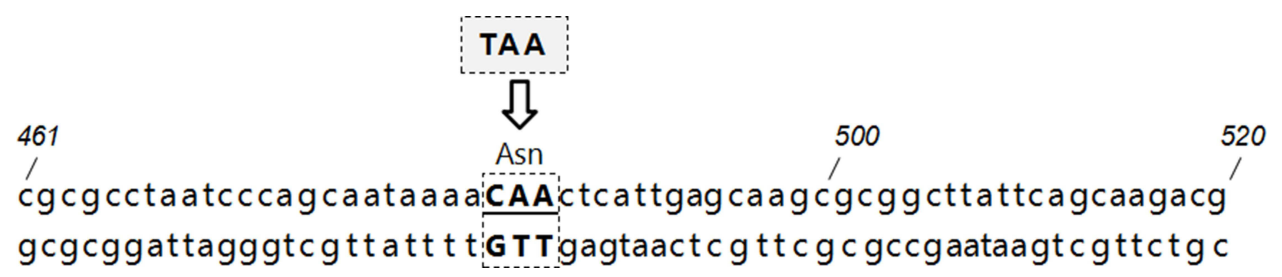

Figure 2 The mutation of the internal stop codons of the functional class 2 integrons: the stop codons in these intl 2 genes were mutated from TAA to the glutamine codon CAA. The position of several nucleotides is numbered (italics).

\section{Discussion}

Class 1 integrons are the most clinically present, most closely associated with antibiotics, and have been most intensively studied. Class 2 integrons, however, are often underappreciated and less studied because of the presence of a stop codon (TAA) on the integrase gene, making it non-functional to integrate, recombine, and cleave the drug resistance cassette. Márquez et $\mathrm{al}^{10}$ identified a specific class 2 integron in a strain of E. coli mediating urinary tract infections and genetically analyzed a sequence shift from the previous termination codon (TAA) to the current glutamyl codon (CAA), and this was the first potentially functional class 2 integron identified from a human pathogen. Wei et al ${ }^{11}$ also found a special class 2 integron strain in the Proteus integron experiment. Gene sequencing revealed that the integrase gene in this strain is consistent with that reported by Márquez, which was able to catalyze and cut the gene cassette. Class 2 integron with full expression ability is called a functional class 2 integron. Current studies on integrons have focused on the interactive relationship between integrase and gene cassette, the relationship between multi-drug resistant bacteria and integron resistance gene cassette, ${ }^{13,16,17}$ effects of different types of promoters causing the expression of integrase gene cassette, ${ }^{18,19}$ and effects of various factors on integron regulatory mechanisms, ${ }^{20}$ but little is known about functional class 2 integrons.

The screening of the integrons of this clinically isolated P. mirabilis showed that among the 150 strains, $20 \%$ were positive for class 1 integrons, $14.7 \%$ were positive for class 2 integrons, $26 \%$ carrying class 1 and class 2 integrons, while class 3 integrons were not detected. The high proportion of $P$. mirabilis-carrying integrons in this study was consistent with other reports, while the carrying rate of class 2 integrons is significantly higher. ${ }^{21,22}$ Six types of resistant gene cassettes were detected in the variable region of class 1 integron-positive strains, mainly dfrA32-aadA2, dfrA32-ereA1aadA2, aadB-aadA2, while the resistance gene cassette detected in the variable region of class 2 integron-positive strains (including three functional class 2 integron-positive strains) was mainly dfrA1-sat2-aadA1. These gene cassettes mediate the host bacterial resistance to trimethoprim, erythromycin, streptomycin, and aminoglycoside, conforming to related reports. ${ }^{11}$ The function of the four ORFs at the end of gene cassette variable regions of class 2 integron remains unclear, We found that class 2 integrons carried a much lower variety of drug resistance gene cassettes than class 1 integrons, probably because of the presence of a stop codon in the reading frame of class 2 integrase genes, impeding it from synthesizing full-length integrase proteins. Thus, class 2 integrases cannot cut and integrate gene cassettes, leading to the singularity of variable region gene cassettes of class 2 integrons. The resistance phenotype analysis further showed that compared to integrase-negative strains, the variable region carrying resistance genes of integrase-positive strains had a

Table 4 Three Functional Class 2 Integron-Related Drug Resistance Genes and Phenotypes

\begin{tabular}{|c|c|c|c|c|c|c|c|c|}
\hline \multirow[t]{2}{*}{ Strains } & \multirow[t]{2}{*}{ intll } & \multirow{2}{*}{$\begin{array}{c}\text { Variable Region of Class } \\
\text { I Integron }\end{array}$} & \multirow[t]{2}{*}{ int/2 } & \multirow{2}{*}{$\begin{array}{c}\text { Variable Region of Class } \\
2 \text { Integron }\end{array}$} & \multicolumn{4}{|c|}{ Susceptibility to Antimicrobial Drugs } \\
\hline & & & & & Amikacin & Gentamicin & Tobramycin & $\begin{array}{l}\text { SMZ- } \\
\text { TMP }\end{array}$ \\
\hline 5 th & - & ND & + & $d f r A I$-sat2-aadAl & $S$ & $S$ & $S$ & $\mathrm{R}$ \\
\hline 30th & + & dfrA32-aadA2 & + & dfrAl-sat2-aadAI & $S$ & $\mathrm{R}$ & $\mathrm{R}$ & $\mathrm{R}$ \\
\hline 33th & - & ND & + & dfrAl-sat2-aadAl & S & $\mathrm{R}$ & S & $\mathrm{R}$ \\
\hline
\end{tabular}

Abbreviation: ND, not detected. 


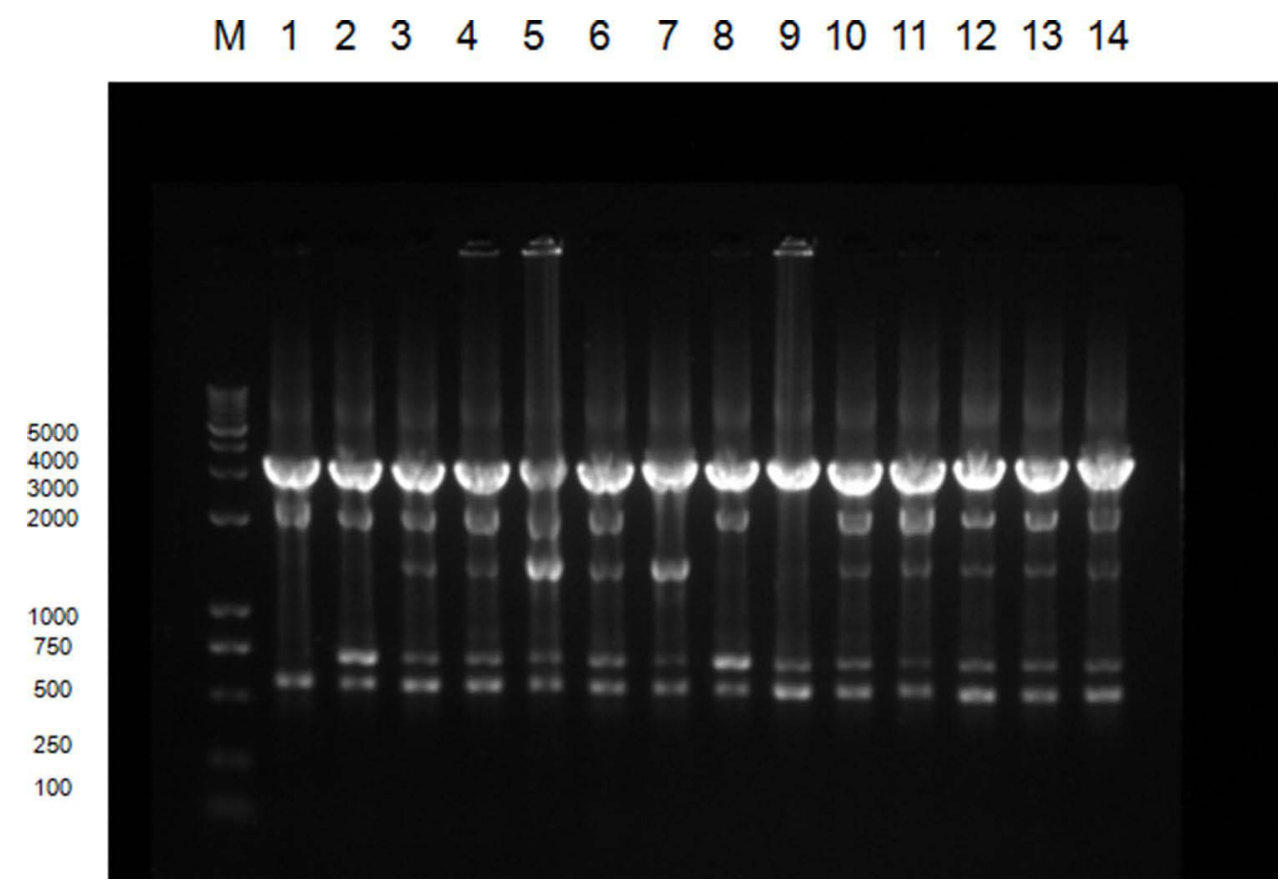

Figure 3 ERIC-PCR typing map of some class 2 integron-positive strains:M represents DNA Marker. Lane 1 is a functional class 2 integron-positive quality control strain; lanes 2 4 are functional class 2 integron-positive strains; lanes 5 14 are class 2 integron-positive strains; lanes 3 6 and 10 14 are type A strains, lanes 2 and 8 are of type B, lane 7 is the type $C$ and lane 9 is a type $D$ strain.

higher rate of resistance to gentamicin, tobramycin, and cotrimoxazole. The rate of resistance to some antimicrobial drugs was also significantly different among strains carrying different integrons. These findings underscored the important role of integrons in bacterial drug resistance and the varying abilities of different integrons in mediating drug resistance in host bacteria, which might be a result of the gene cassette type carried by integrons, their gene expression status, and differences in promoter strength. ${ }^{23,24}$

The expression of gene cassettes depends on the promoter Pc. In class 1 integrons, the Pc promoter is located mainly in the integrase-encoding sequence, ${ }^{25}$ and the expression of drug-resistant integrase involves four kinds of promoters $(\mathrm{PcS}, \mathrm{PcW}, \mathrm{PcH} 1$, and $\mathrm{PcH} 2)$, which is affected by the $\mathrm{P} 2$ promoter. The expression of the gene cassette is also positively correlated with promoter strength and negatively with the distance from the promoter. ${ }^{26,27}$ However, in class 2 integrons, the Pc promoter was not detected in the integrase gene because a part of the integron recombination site region can achieve gene cassette expression. ${ }^{8}$ In this study, sequencing of functional class 2 integron promoters revealed that the region from integron recombination site attI2 to downstream $317 \mathrm{bp}$ was relatively conserved. Four types of Pc promoter sequences found were Pc2D, Pc2A, Pc2B, and Pc2C, identical to the promoter sequences of other class 2 integronpositive strains. This finding is highly consistent with Thomas's model of class 2 integron promoters, ${ }^{15}$ whose studies have confirmed that active regions of class 2 integron promoter are mainly Pc2A and Pc2B, and unlike class 1 integrons, class 2 integron promoters are not regulated by the bacterial SOS system.

The integrase sequence of functional class 2 integrons of the three screened strains was consistent with that reported by Quhao Wei, whose team examined the integration and excision effects of gene cassettes using quantitative real-time PCR for all five functional class 2 integrons submitted in the INTEGRALL database, and found only little differences in these effects. ${ }^{28}$ Our study showed that the sequence of functional class 2 integron promoters is identical to that of other class 2 integrases in the same cohort, indicating that increased integrase activity did not alter promoter sequence and activity of the attI2 region at the recombination site. Whole gene sequencing, as well as upstream and downstream gene analysis, showed the presence of all class 2 integrons in this screening on the $\operatorname{Tn} 7$ transposon of the host bacterial chromosome, which was significantly different from the transmission mechanism of class 1 integrons present in plasmids for propagation. Based on the location of class 2 integron, it can be speculated that the bacterial genome stability limits 
the activity of class 2 integrases, which largely explains why the sequence of class 2 integrases is relatively conserved and singular. Further studies are needed to investigate whether functional class 2 integrases present on the chromosome restore the integration and cutting activity in the wild environment and the mechanism and reason for the presence of all class 2 integrases on the $\mathrm{Tn} 7$ transposon of the host chromosome. The deeper reasons for differences in classes and functions of integrons may be due to the natural selection of bacteria in the process of transmission and evolution.

The ERIC-PCR homology analysis showed that most of the 61 clinically isolated P. mirabilis strains had high genotyping homology, suggesting that there may be clonal transmission in the hospital. Among the three functional class 2 integron-positive strains, two were highly homologous, with the same genotype as other class 2 integron-positive strains screened in this study. Therefore, class 2 integrons may be affected by external environmental factors or regulated by bacterial genome during transmission, resulting in the stop codon mutation, thereby restoring integrase activity, transforming into functional class 2 integrons, to adapt to the environmental changes and survival.

\section{Conclusions}

Functional class 2 integrons, a special form of integrons, currently have a low clinical distribution and are occasionally isolated in clinical strains such as those of $P$. mirabilis. Compared to class 1 integrons, specific localization of class 2 integrons on the host bacterium chromosome and its lower intensity $\mathrm{P}_{\mathrm{C}}$ promoters result in a reduced ability to integrate, cut, and express drug resistance gene cassette in variable regions. However, the resurrection of gene mutations in functional class 2 integrons could be also explained by the fact that bacteria are adapting to the environment during transmission and evolution. Furthermore, functional class 2 integrons also cause clonal transmission among bacteria.

\section{Abbreviations}

P. mirabilis, Proteus mirabilis; PCR, polymerase chain reaction; ERIC-PCR, enterobacterial repetitive intergenic consensus polymerase chain reaction; SMZ/TMP, sulfamethoxazole/trimethoprim; ORFs, open reading frames.

\section{Ethical Statement}

All clinical data and related data of this case have been approved by the hospital ethics committee and informed consent of the patient.

\section{Author Contributions}

All authors made a significant contribution to the work reported, whether that is in the conception, study design, execution, acquisition of data, analysis and interpretation, or in all these areas; took part in drafting, revising or critically reviewing the article; gave final approval of the version to be published; have agreed on the journal to which the article has been submitted; and agree to be accountable for all aspects of the work.

\section{Funding}

This study was supported by the research grants from Ningbo Municipal Natural Science Fund (No. 2018 A610402).

\section{Disclosure}

The authors report no conflicts of interest in this work.

\section{References}

1. Faghri J, Nouri S, Jalalifar S, et al. Investigation of antimicrobial susceptibility, class I and II integrons among Pseudomonas aeruginosa isolates from hospitalized patients in Isfahan, Iran. BMC Res Notes. 2018;11:806. doi:10.1186/s13104-018-3901-9

2. Halaji M, Rezaei A, Zalipoor M, et al. Investigation of class I, II, and III integrons among Acinetobacter baumannii isolates from hospitalized patients in Isfahan, Iran. Oman Med J. 2018;33:37-42. doi:10.5001/omj.2018.07

3. Halaji M, Feizi A, Mirzaei A, et al. The global prevalence of class 1 integron and associated antibiotic resistance in Escherichia coli from patients with urinary tract infections, a systematic review and meta-analysis. Microb Drug Resist. 2020;26:1208-1218. doi:10.1089/mdr.2019.0467

4. Jechalke S, Schreiter S, Wolters B, et al. Widespread dissemination of class 1 integron components in soils and related ecosystems as revealed by cultivation -independent analysis. Front Microbiol. 2013;4:420. doi:10.3389/fmicb.2013.00420 
5. Rowe-Magnus DA, Mazel D. The role of integrons in antibiotic resistance gene capture. Int J Med Microbiol. 2002;292:115-125. doi:10.1078/ 1438-4221-00197

6. Ploy MC, Denis F, Courvalin P, et al. Molecular Characterization of Integrons in Acinetobacter baumannii: description of a Hybrid Class 2 Integron. Antimicrob Agents Chemother. 2000;44:2684-2688. doi:10.1128/AAC.44.10.2684-2688.2000

7. Japoni-Nejad A, Farshad S, van Belkum A, et al. Novel cassette array in a class 1 integron in clinical isolates of Acinetobacter baumannii from central Iran. Int J Med Microbiol. 2013;303:645-650.

8. Hansson K, Sundstrom L, Pelletier A, et al. IntI2 integron integrase in Tn 7. J Bacteriol. 2002;184:1712. doi:10.1128/JB.184.6.1712-1721.2002

9. Ramírez MS, Quiroga C, Centrón D. Novel rearrangement of a class 2 integron in two non-epidemiologically related isolates of Acinetobacter baumannii. Antimicrob Agents Chemother. 2005;49:5179-5181. doi:10.1128/AAC.49.12.5179-5181.2005

10. Márquez C, Labbate M, Ingold AJ, et al. Recovery of a functional class 2 integron from an Escherichia coli strain mediating a urinary tract infection. Antimicrob Agents Chemother. 2008;52:4153-4154. doi:10.1128/AAC.00710-08

11. Wei Q, Hu Q, Li S, et al. A novel functional class 2 integron in clinical Proteus mirabilis isolates. J Antimicrob Chemother. $2014 ; 69: 973-976$. doi:10.1093/jac/dkt456

12. Weinstein MP, Lewis JS. Commentary: the Clinical and Laboratory Standards Institute (CLSI) subcommittee on antimicrobial susceptibility testing: background, organization, functions, and processes. J Clin Microbiol. 2020;58(3). doi:10.1128/JCM.01864-19

13. Su J, Shi L, Yang L, et al. Analysis of integrons in clinical isolates of Escherichia coli in China during the last six years. FEMS Microbiol Lett. 2006;254:75-80. doi:10.1111/j.1574-6968.2005.00025.x

14. Corvec S, Prodhomme A, Giraudeau C, et al. Most Escherichia coli strains overproducing chromosomal AmpC -lactamase belong to phylogenetic group A. J Antimicrob Chemother. 2007;60:872-876. doi:10.1093/jac/dkm284

15. Jové T, Da Re S, Tabesse A, et al. Gene expression in class 2 integrons is SOS-independent and involves two Pc promoters. Front Microbiol. 2017;8:1499. doi:10.3389/fmicb.2017.01499

16. Shi L, Zheng M, Xiao Z, et al. Unnoticed spread of class 1 integrons in gram-positive clinical strains isolated in Guangzhou, China. Microbiol Immunol. 2006;50:463-467. doi:10.1111/j.1348-0421.2006.tb03815.x

17. Panopoulou M, Alepopoulou E, Ikonomidis A, et al. Emergence of VIM-12 in Enterobacter cloacae. J Clin Microbiol. 2010;48:3414-3415. doi:10.1128/JCM.00843-10

18. Soufi L, Sáenz Y, Vinué L, et al. Characterization of Pc promoter variants of class 1 integrons in Escherichia coli isolates from poultry meat. Foodborne Pathog Dis. 2013;10:1075-1077. doi:10.1089/fpd.2013.1542

19. Wei Q, Jiang X, Li M, et al. Transcription of integron-harboured gene cassette impacts integration efficiency in class 1 integron. Mol Microbiol. 2011;80:1326-1336. doi:10.1111/j.1365-2958.2011.07648.x

20. Guérin E, Jové T, Tabesse A, et al. High-level gene cassette transcription prevents integrase expression in class 1 integrons. $J$ Bacteriol. 2011;193:5675-5682. doi:10.1128/JB.05246-11

21. Wu K, Wang F, Sun J, et al. Class 1 integron gene cassettes in multidrug-resistant Gram-negative bacteria in southern China. Int J Antimicrob Agents. 2012;40:264-267. doi:10.1016/j.ijantimicag.2012.05.017

22. El-Sokkary MA, El-Sokkary MMA, Aabed R, et al. Identification, antibiotic resistance and distribution of different classes of integrons among proteus species isolated from different sources in Dakahleia and Damietta Egyptian Governorates. Afri J Microbiol Res. 2015;9:1312-1321. doi:10.5897/AJMR2015.7486

23. Xiao L, Wang X, Kong N, et al. Polymorphisms of gene cassette promoters of the class 1 integron in clinical proteus isolates. Front Microbiol. 2019;10:790. doi:10.3389/fmicb.2019.00790

24. Liu M, Ma J, Jia W, et al. Antimicrobial resistance and molecular characterization of gene cassettes from class 1 integrons in Pseudomonas aeruginosa strains. Microb Drug Resist. 2020;26:670-676. doi:10.1089/mdr.2019.0406

25. Collis CM, Hall RM. Expression of antibiotic resistance genes in the integrated cassettes of integrons. Antimicrob Agents Chemother. 1995;39:155162. doi:10.1128/AAC.39.1.155

26. Vinué L, Jové T, Torres C, et al. Diversity of class 1 integron gene cassette Pc promoter variants in clinical Escherichia coli strains and description of a new P2 promoter variant. Int J Antimicrob Agents. 2011;38:526-529. doi:10.1016/j.ijantimicag.2011.07.007

27. Wei Q, Jiang X, Li M, et al. Diversity of gene cassette promoter variants of class 1 integrons in uropathogenic Escherichia coli. Curr Microbiol. 2013;67:543-549. doi:10.1007/s00284-013-0399-1

28. Wang X, Kong N, Cao M, et al. Comparison of class 2 integron integrase activities. Curr Microbiol. 2021;78:967-978. doi:10.1007/s00284-021$02352-9$

Infection and Drug Resistance

Dovepress

\section{Publish your work in this journal}

Infection and Drug Resistance is an international, peer-reviewed open-access journal that focuses on the optimal treatment of infection (bacterial, fungal and viral) and the development and institution of preventive strategies to minimize the development and spread of resistance. The journal is specifically concerned with the epidemiology of antibiotic resistance and the mechanisms of resistance development and diffusion in both hospitals and the community. The manuscript management system is completely online and includes a very quick and fair peer-review system, which is all easy to use. Visit http://www.dovepress.com/testimonials.php to read real quotes from published authors.

Submit your manuscript here: https://www.dovepress.com/infection-and-drug-resistance-journal 